\title{
Efficacy of Biofeedback for Medical Conditions: an Evidence Map
}

\author{
Karli Kondo, $P h D, M A^{1,2}{ }^{\oplus}$, Katherine M. Noonan, $P h D^{3}$, Michele Freeman, $M P H^{1}$, \\ Chelsea Ayers, $B A^{7}$, Benjamin J. Morasco, $P h D^{4,5}$, and Devan Kansagara, MD, MCR ${ }^{1,4,6}$
}

'Evidence Synthesis Program, VA Portland Health Care System, Portland, OR, USA; ${ }^{2}$ Research Integrity Office, Oregon Health \& Science University, Portland, OR, USA; ${ }^{3}$ Physical Medicine and Rehabilitation, VA Portland Health Care System, Portland, OR, USA; ${ }^{4}$ Center to Improve Veteran Involvement in Care, VA Portland Health Care System, Portland, OR, USA; ${ }^{5}$ Department of Psychiatry, Oregon Health \& Science University, Portland, OR, USA; ${ }^{6}$ Department of Medicine, Oregon Health \& Science University, Portland, OR, USA.

BACKGROUND: Biofeedback is increasingly used to treat clinical conditions in a wide range of settings; however, evidence supporting its use remains unclear. The purpose of this evidence map is to illustrate the conditions supported by controlled trials, those that are not, and those in need of more research.

METHODS: We searched multiple data sources (MEDLINE, PsycINFO, CINAHL, Epistemonikos, and EBM Reviews through September 2018) for good-quality systematic reviews examining biofeedback for clinical conditions. We included the highest quality, most recent review representing each condition and included only controlled trials from those reviews. We relied on quality ratings reported in included reviews. Outcomes of interest were condition-specific, secondary, and global health outcomes, and harms. For each review, we computed confidence ratings and categorized reported findings as no effect, unclear, or insufficient; evidence of a potential positive effect; or evidence of a positive effect. We present our findings in the form of evidence maps.

RESULTS: We included 16 good-quality systematic reviews examining biofeedback alone or as an adjunctive intervention. We found clear, consistent evidence across a large number of trials that biofeedback can reduce headache pain and can provide benefit as adjunctive therapy to men experiencing urinary incontinence after a prostatectomy. Consistent evidence across fewer trials suggests biofeedback may improve fecal incontinence and stroke recovery. There is insufficient evidence to draw conclusions about effects for most conditions including bruxism, labor pain, and Raynaud's. Biofeedback was not beneficial for urinary incontinence in women, nor for hypertension management, but these conclusions are limited by small sample sizes and methodologic limitations of these studies.

DISCUSSION: Available evidence suggests that biofeedback is effective for improving urinary incontinence after prostatectomy and headache, and may provide benefit for fecal

SR Registration: NA (evidence maps are not eligible for PROSPERO reaistration)

Electronic supplementary material The online version of this article (https://doi.org/10.1007/s11606-019-05215-z) contains supplementary material which is available to authorized users.

Received May 20, 2019

Accepted July 12, 2019

Published online August 14, 2019 incontinence and balance and stroke recovery. Further controlled trials across a wide range of conditions are indicated.

KEYWORDS: biofeedback; neurofeedback; complementary and alternative medicine (CAM); evidence map.

J Gen Intern Med 34(12):2883-93

DOI: $10.1007 / \mathrm{s} 11606-019-05215-Z$

(C) Society for General Internal Medicine (This is a U.S. government work and not under copyright protection in the U.S.; foreign copyright protection may apply) 2019

\section{INTRODUCTION}

Biofeedback emerged as a field of study in the 1960s, and in the last few decades, it has increasingly been used as a complementary or alternative treatment for a wide range of clinical conditions and symptom reduction. Biofeedback uses instruments to measure and provide real-time feedback on patients' physiological responses in order to assist patients in learning to change those responses. ${ }^{1}$ The most common types of measurements are muscle activity, heart rate function and variability, respiration, blood pressure and flow, brainwaves, skin temperature, and electrodermal (sweat gland) activity. ${ }^{1,2}$ Receipt of this feedback increases patients' awareness of these physiological processes. Ideally, when this awareness is paired with interventions to change behavior, thoughts, or emotions, a beneficial change in the physiological process occurs. The ultimate goal is for this change to eventually be maintained in all settings and without the need for equipment. ${ }^{3}$

The challenge of treating chronic symptomatic conditions, and the potential harms associated with pharmacotherapies for these conditions, has prompted increasing interest in effective nonpharmacologic treatment alternatives such as biofeedback. One of the challenges in considering the therapeutic role of treatments like biofeedback that have not been well integrated into allopathic health care systems is that the evidence base is scattered across numerous conditions. The Association for Applied Psychophysiology and Biofeedback (AAPB) provides clinical guidelines for the use of biofeedback for a diverse list of conditions. ${ }^{4}$ The most recent guidelines ${ }^{2}$ were published in 2016 and considered studies ranging from single-subject case studies to 
randomized controlled trials (RCTs). To date, no review has examined the use of biofeedback across conditions.

Evidence mapping is a recently developed methodology to provide a high-level overview of the state of evidence for a given intervention across a diverse set of conditions. This "lay of the land" of the evidence can be useful to health systems, policymakers, researchers, and clinicians who would like to prioritize the most promising areas and conditions for treatment and further research. Although standardized definitions and methodology are still being established, they generally include a systematic search of a broad field of research and a userfriendly, often visual, representation of a body of literature. ${ }^{5}$

This evidence map was conducted to understand and illustrate current empirical data from controlled trials about the benefits and harms of biofeedback for different medical conditions. We will broadly identify the strengths, weaknesses, and gaps in the evidence base, and highlight areas for future research.

\section{METHODS}

We systematically reviewed systematic reviews and metaanalyses examining biofeedback as a primary or adjunctive intervention and created maps illustrating the evidence. This was part of a larger report for the Department of Veterans Affairs that also summarized the evidence base for guided imagery and hypnosis for medical conditions. ${ }^{6}$ Our approach was guided by an analytic framework (Online Appendix Fig. 1), and our protocol was developed using established reporting standards.

\section{Data Sources and Searches}

To identify relevant systematic reviews/meta-analyses, we searched MEDLINE, PsycINFO, CINAHL, Epistemonikos, and EBM Reviews Cochrane Database of Systematic Reviews from database inception to September 2018 (see Online Appendix Table 1). In addition, we reviewed the bibliographies of relevant reviews of reviews, searched the registry PROSPERO for completed reviews, and queried subject matter experts. For all methods applicable to evidence maps, we followed the PRISMA guidelines. ${ }^{7}$

\section{Study Selection and Assessment of Study Quality}

Using pre-specified inclusion criteria (Online Appendix Tables 2 and 3), two investigators independently evaluated the titles, abstracts, and full text of all potentially relevant systematic reviews for inclusion. All discordant results were resolved through consensus or consultation with a third reviewer. Eligible systematic reviews met all of the following quality criteria: (1) clearly reported their search strategy and inclusion criteria; (2) performed a comprehensive search of at least two electronic databases; and (3) assessed the methods and potential risk of bias in the included trials using validated criteria. ${ }^{8}$ We included only systematic reviews that specifically examined the use of biofeedback for adults with a specific clinical condition. From the systematic reviews meeting our eligibility criteria, we identified a single review to represent each clinical condition (based on recency, size, and overall quality). From each included systematic review, we included only controlled trials of adults treated with biofeedback alone or in combination with other interventions. We relied on the quality assessments reported in the included systematic reviews.

\section{Data Abstraction}

Data from included systematic reviews were abstracted by one investigator and confirmed by a second. From each systematic review, we abstracted data related to the biofeedback intervention and any concurrent interventions, clinical condition, number of studies and subjects included, and relevant findings for each outcome of interest.

\section{Evidence Maps}

We created maps to illustrate the evidence from controlled trials published in good-quality systematic reviews examining the use of biofeedback for different medical conditions. For each condition, we categorized outcomes into the following categories: (1) diagnosis-related; (2) secondary-specific symptom outcomes that are not directly related to the condition (e.g., anxiety in patients with headache); (3) global health (e.g., quality of life, self-efficacy); and (4) harms. For each condition and outcome category, we recorded whether the clinical trials in the review found evidence of no effect, unclear, or insufficient evidence, evidence of a potential positive effect, or evidence of a positive effect. If a review reported multiple outcomes within the same category (e.g., depression and anxiety), we classified the outcome as potentially positive if there was at least one clear finding of benefit (see Online Appendix Table 4 for a detailed description of our methods).

Guided by methods employed in prior evidence maps, ${ }^{5}$ and established strength of evidence criteria, ${ }^{9}$ we developed an estimate of confidence (insufficient, low, moderate, high) for each outcome category by condition (represented by bubble size on the map). Confidence estimates were based on the following: (1) sample size; (2) consistency; (3) directness; and (4) overall study quality, as reported by the systematic review (see Online Appendix Table 4 for our scoring criteria and descriptions). As with previously published evidence maps, the confidence estimate allows for an additional dimension on which to broadly assess gaps in evidence, rather than reflecting an assessment of the strength of evidence.

\section{RESULTS}

We reviewed 2533 titles and abstracts, and the full text of 229 systematic reviews. We identified 16 systematic reviews examining the effectiveness of biofeedback on unique primary/ 
diagnosis-related outcomes, secondary outcomes, and global outcomes (see Online Appendix Fig. 2 for the literature flow diagram). The number of trials in the systematic reviews ranged from 1 (knee osteoarthritis) ${ }^{10}$ to 94 (headache), ${ }^{11}$ and the sample size of the trials ranged from $56^{10}$ to over $3500^{11}$ (see Fig. 1). Biofeedback modalities varied both within and by condition, as did the use of adjunctive interventions (see Table 1). Across systematic reviews, all 16 examined primary diagnosis-related outcomes, six also evaluated secondary outcomes, six assessed global outcomes, and only one reported harms (see Table 2).

For five conditions (fecal incontinence, ${ }^{12}$ urinary incontinence in women,${ }^{13}$ dysphagia, ${ }^{14}$ stroke,${ }^{15}$ and Bell's palsy ${ }^{16}$ ), systematic reviews specifically examined biofeedback as an adjunct to another intervention. Five reviews examined the effectiveness of biofeedback for conditions (sleep bruxism, ${ }^{17}$ chronic idiopathic constipation, ${ }^{18}$ knee osteoarthritis, ${ }^{10}$ balance/gait training, ${ }^{19}$ and intradialytic hypotension ${ }^{20}$ ) independent of other interventions. For all other conditions, systematic reviews included both studies examining biofeedback alone and as an adjunct to another intervention (Table 1). ${ }^{11,21-24}$

We found high-confidence evidence that biofeedback is effective for reducing the frequency, duration, and intensity of migraine and tension-type headaches. There is also moderate-confidence evidence of benefit on secondary outcomes of headaches such as medication intake, muscle tension, anxiety, and depression. There is limited evidence supporting the benefit of biofeedback for improving global self-efficacy in patients with migraine or tension-type headaches. ${ }^{11}$

We found high-confidence evidence that biofeedback as an adjunctive treatment for pelvic floor muscle training (PFMT) can result in both immediate- and long-term improvements in urinary incontinence for men after a prostatectomy as compared with PFMT alone. The addition of biofeedback also had

3500

3000

2500

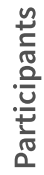

2000

1500

1000

500

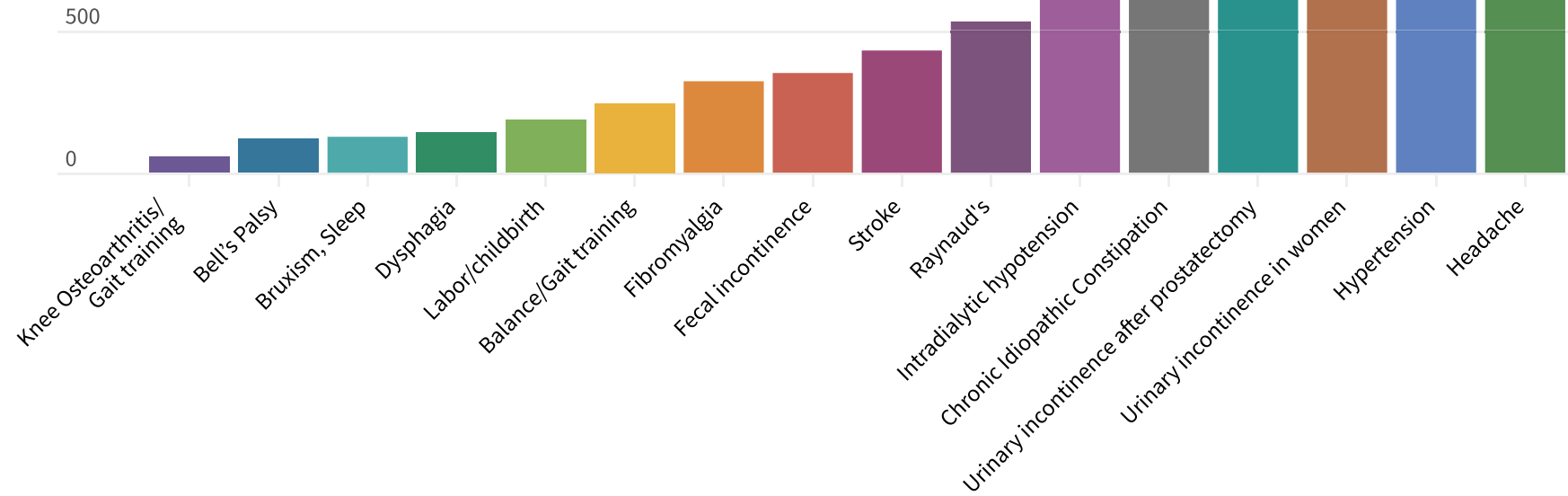

Figure 1 Number of participants by condition. 
Table 1 Type of Biofeedback and Adjunctive Interventions by Condition

\begin{tabular}{|c|c|c|}
\hline$\overline{\text { Condition }}$ & Biofeedback techniques used & Adjunctive therapies \\
\hline Sleep bruxism ${ }^{17}$ & Contingent electrical stimulation & - \\
\hline $\begin{array}{l}\text { Urinary incontinence after } \\
\text { prostatectomy }^{21}\end{array}$ & Biofeedback-assisted pelvic floor muscle training & Varied - electrical stimulation \\
\hline Chronic idiopathic constipation ${ }^{18}$ & $\begin{array}{l}\text { EMG biofeedback, balloon sensory biofeedback, } \\
\text { manometry biofeedback }\end{array}$ & - \\
\hline Fecal incontinence $^{12}$ & EMG biofeedback, balloon sensory biofeedback & With electrical stimulation \\
\hline Urinary incontinence (women) ${ }^{13}$ & $\begin{array}{l}\text { EMG, vaginal and/or anal squeeze pressure, } \\
\text { ultrasound }\end{array}$ & With pelvic floor muscle training \\
\hline Hypertension $^{22}$ & $\begin{array}{l}\text { Blood pressure biofeedback, indirect biofeedback, } \\
\text { direct biofeedback }\end{array}$ & $\begin{array}{l}\text { Varied-relaxation, meditation, imagery, } \\
\text { inner quality management }\end{array}$ \\
\hline Dysphagia $^{14}$ & $\begin{array}{l}\text { sEMG, accelerometry, tongue manometry, video } \\
\text { endoscopy, respiratory plethysmography, external } \\
\text { laryngeal manometry }\end{array}$ & With swallow therapy \\
\hline Fibromyalgia $^{23}$ & $\begin{array}{l}\text { EMG biofeedback, EEG feedback, LENS, } \\
\text { SMR training }\end{array}$ & Varied-PMR \\
\hline $\begin{array}{l}\text { Knee osteoarthritis/gait } \\
\text { retraining }\end{array}$ & Visual, haptic (not specified) & - \\
\hline Stroke $^{15^{\circ}}$ & $\begin{array}{l}\text { Weight distribution from a force platform or sensor, } \\
\text { muscle activity from EMG, linear gait parameters } \\
\text { from foot sensors, joint angle from a goniometer }\end{array}$ & $\begin{array}{l}\text { With usual therapy including therapist } \\
\text { communication }\end{array}$ \\
\hline Headache $^{11}$ & $\begin{array}{l}\text { TEMP biofeedback, TEMP + EMG biofeedback, } \\
\text { EMG biofeedback, BVP biofeedback, EEG } \\
\text { biofeedback, GSR biofeedback }\end{array}$ & Varied-relaxation \\
\hline Balance/gait training ${ }^{19}$ & Wearable plantar pressure sensors, IMU & - \\
\hline Labor pain ${ }^{24}$ & EMG, skin conductance biofeedback & Varied-relaxation, PMR, Lamaze \\
\hline Bell's palsy ${ }^{16}$ & EMG, biofeedback rehabilitation & $\begin{array}{l}\text { With mime therapy. Other therapies } \\
\text { varied-facial expression exercises, } \\
\text { lip movement without eye closure }\end{array}$ \\
\hline Raynaud's ${ }^{26}$ & Thermal biofeedback, thermal feedback + EMG & Varied - autogenic training, relaxation \\
\hline Intradialytic hypotension ${ }^{20}$ & $\begin{array}{l}\text { Biofeedback hemodialysis: BVM with dialysate } \\
\text { conductivity control, BVM with plasma } \\
\text { conductivity control }\end{array}$ & - \\
\hline
\end{tabular}

BFB biofeedback, BVM blood volume monitoring, BVP blood volume pulse, EEG electroencephalograph, EMG electromyograph, IMU inertial measurement units, GSR galvanic skin response, LENS low-intensity neurofeedback system, PMR progressive muscle relaxation, sEMG surface electromyography, SMR sensorimotor rhythm, TEMP peripheral temperature feedback

a positive effect on quality of life (moderate confidence). ${ }^{21}$ For both short- and long-term lower limb activity improvements after stroke (e.g., standing, walking), there is (moderateconfidence) evidence that the addition of biofeedback to usual therapy is more effective than usual therapy alone. ${ }^{25}$ Finally, for fecal incontinence, electrical stimulation with biofeedback is more effective than electrical stimulation alone (moderate confidence; Fig. 2 and Table 3). ${ }^{12}$
We identified limited (low-confidence) evidence that biofeedback hemodialysis can result in lower rates of mortality and intradialytic hypotension (IDH) in patients undergoing hemodialysis who are experiencing chronic fluid overload or symptomatic IDH. ${ }^{20}$ In patients with fibromyalgia, electromyograph (EMG), but not electroencephalograph (EEG) biofeedback has the potential to improve short- and long-term pain (but not quality of life or

Table 2 Number of Studies and Participants by Condition and Outcome

\begin{tabular}{|c|c|c|c|c|c|}
\hline \multirow[b]{2}{*}{ Condition } & \multirow[b]{2}{*}{ Total no. of studies $(N)$} & \multicolumn{4}{|c|}{ No. of studies by outcome $(N)$} \\
\hline & & Diagnosis-related & Secondary & Global health & Harms \\
\hline Balance/gait training ${ }^{19}$ & $8(243)$ & $8(243)$ & $3(99)$ & $2(75)$ & - \\
\hline Bell's palsy $^{16}$ & $4(118)$ & $4(118)$ & - & - & - \\
\hline Bruxism, sleep $^{17}$ & $6(126)$ & $6(126)$ & $1(12)$ & - & - \\
\hline Chronic idiopathic constipation $^{18}$ & $17(931)$ & $17(931)$ & - & - & - \\
\hline Dysphagia $^{14}$ & $5(141)$ & $5(141)$ & - & - & - \\
\hline Fecal incontinence ${ }^{12}$ & $12(350)$ & $12(350)$ & - & - & - \\
\hline Fibromyalgia $^{23}$ & $7(580)$ & $7(289)$ & $5(295)$ & $4(163)$ & $3(185)$ \\
\hline Hypertension $^{22}$ & $36(1660)$ & $36(1660)$ & - & - & - \\
\hline Intradialytic hypotension $^{20}$ & $8(716)$ & $6(266)$ & $6(266)$ & $3(270)$ & - \\
\hline Knee osteoarthritis/gait training ${ }^{10}$ & $1(56)$ & $1(56)$ & - & - & - \\
\hline Pain, headache ${ }^{11}$ & $94(3500)$ & $62(1285)$ & Unclear & Unclear & - \\
\hline Labor/childbirth ${ }^{24}$ & $4(186)$ & $4(186)$ & - & - & - \\
\hline Raynaud's 26 & $10(531)$ & $10(531)$ & - & - & - \\
\hline Stroke $^{25}$ & $18(429)$ & $18(429)$ & - & - & - \\
\hline Urinary incontinence after prostatectomy ${ }^{21}$ & $13(1108)$ & $13(1108)$ & - & $5(354)$ & - \\
\hline Urinary incontinence in women $^{13}$ & $24(1583)$ & $5(520)$ & $24(1583)$ & $12(698)$ & - \\
\hline
\end{tabular}




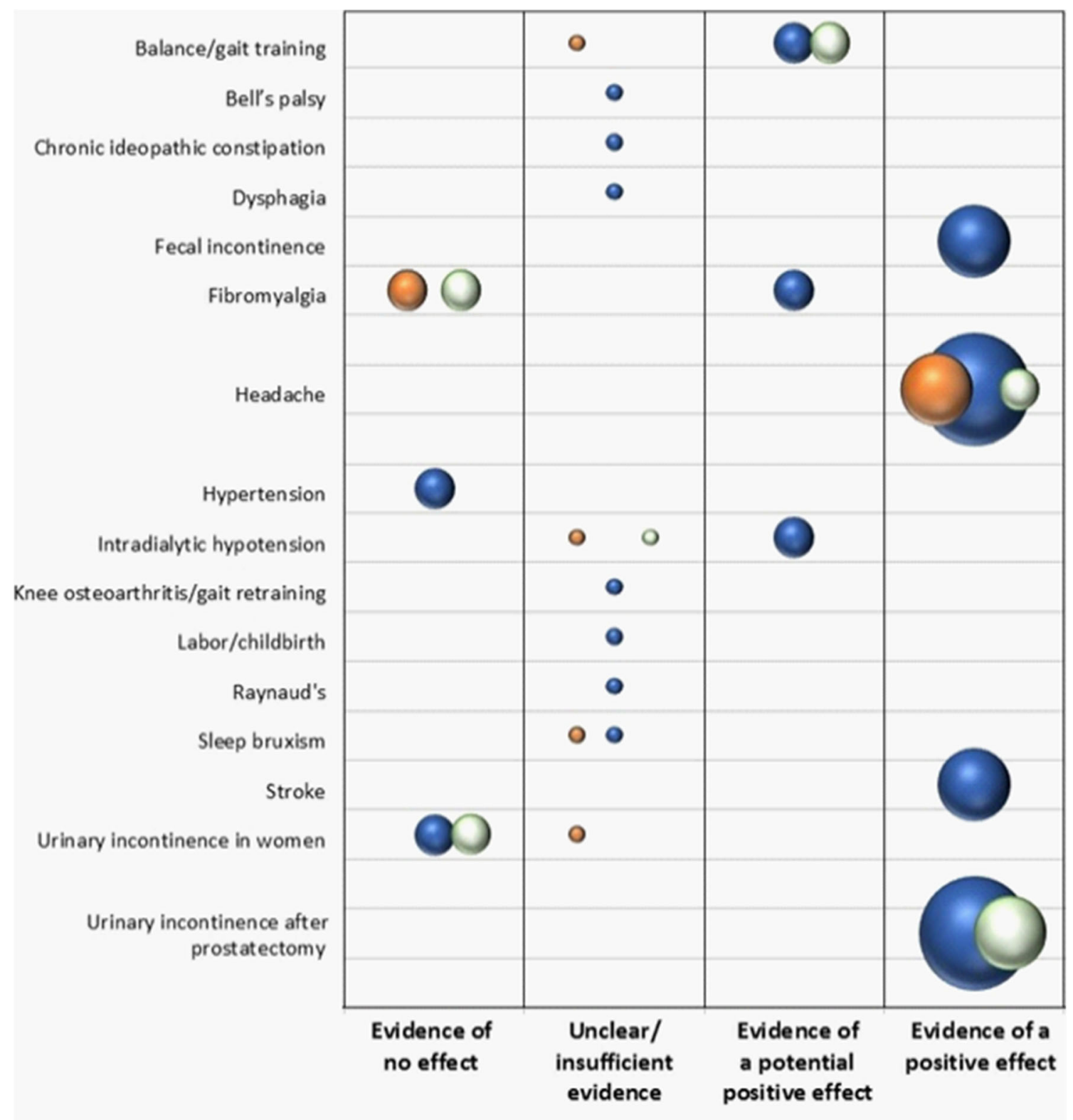

\begin{tabular}{|l|l|}
\hline Outcomes & Levels of confidence \\
Diagnosis-related & High* \\
Slobal & Low \\
\hline
\end{tabular}

Figure 2 Evidence map of biofeedback by condition and outcome category (3D map).

secondary outcomes) ${ }^{23}$ Finally, wearable plantar pressure sensors (which measure the distribution of plantar pressure, usually when standing or moving) may provide better static steady-state balance and health-related quality-of-life outcomes for patients undergoing balance or gait training (Fig. 2 and Table 3). ${ }^{19}$

It is unclear whether biofeedback is effective for the treatment of Bell's palsy, ${ }^{16}$ chronic idiopathic constipation, ${ }^{18}$ dysphagia, ${ }^{14}$ osteoarthritis of the knee, ${ }^{10}$ labor pain, ${ }^{24}$ Raynaud's, ${ }^{26}$ and sleep bruxism. ${ }^{17}$

Limited (low-confidence) evidence suggests that biofeedback provides no benefit for urinary incontinence in women once differences in PFMT programs were controlled for, ${ }^{13}$ nor was it effective for blood pressure control. ${ }^{22}$ Findings related to all other conditions were insufficient, largely due to mixed findings and/or small sample sizes (Fig. 2 and Table 3). 
Table 3 Summary of Findings

\begin{tabular}{|c|c|c|c|}
\hline $\begin{array}{l}\text { Condition/target } \\
\text { population } \\
N \text { controlled trials }(N \\
\text { combined participants) }\end{array}$ & $\begin{array}{ll}\text { Outcomes } & \text { Findings }\end{array}$ & $\begin{array}{l}\text { Summary of } \\
\text { effect }\end{array}$ & $\begin{array}{l}\begin{array}{l}\text { Overall } \\
\text { confidence }\end{array} \\
\end{array}$ \\
\hline
\end{tabular}

Urinary incontinence after $13(N=1108)^{\mathrm{a}}$ related prostatectomy $^{21}$
Urinary incontinence in women $^{13}$

24 trials $(N=1583$

[biofeedback] $)^{\mathrm{b}}$
Objective measurement of urinary incontinence improvement: favors

PFMT + biofeedback (immediate-, intermediate-, and long-term) versus pelvic floor muscle training alone $(P=0.023,0.002$, and 0.017 , respectively)

Subjective measurement of urinary incontinence improvement: favors PFMT + biofeedback (intermediate- and long-term) versus pelvic floor muscle training alone $(P=0.034$ and 0.005 , respectively). There were no significant immediate effects $(P=0.108)$

Secondary

Global

Quality of life: favors PFMT + biofeedback (immediate- and intermediate-term) versus pelvic floor muscle training alone $(P=$ 0.003 and 0.11 , respectively). There was no effect on long-term urinary incontinence $(P=0.080)$

Diagnosisrelated

Self-reported symptomatic cure or improvement:

PFMT + BF versus PFMT (9 RCTs, $N=604)$ : favored PFMT + biofeedback to PFMT alone ( $R R=0.75,95 \%$ CI [0.66 to 0.86$]$ ).

However, there was significant heterogeneity in PFMT and subgroup analyses found no difference between groups between biofeedback and no biofeedback

PFMT versus PFMT + feedback + biofeedback - cure versus no cure ( $1 \mathrm{RCT}, N=152)$ : no difference $(\mathrm{OR}=1.59,95 \%$ CI 0.43 to 5.87 ) PFMT + BF versus PFMT + feedback $(2 \mathrm{RCTs}, N=130):$ no difference

Secondary Number of leakage episodes in $24 \mathrm{~h}$ :

PFMT versus PFMT + feedback + biofeedback - cure versus no cure (1 RCT, $N=152)$ : no difference $(Z=1.04, P=0.30)$.

PFMT + BF versus PFMT + feedback (3 RCTs, $N=267)$ : no

difference

Pelvic floor muscle function:

PFMT versus PFMT + feedback + biofeedback-repetitions, endurance, perineometry, modified Oxford Scale, number of fast contractions ( $1 \mathrm{RCT}, N=152$ ): favored PFMT with feedback and BF group versus PFMT alone.

PFMT + BF versus PFMT + feedback-\% of subjects with increase on EMG assessment, ultrasound displacement, pressure perineometry, digital vaginal palpation, endurance (sitting, standing), amplitude EMG (4 RCTs, $N=180$ ): mixed findings

Frequency of micturition:

PFMT versus PFMT + feedback + biofeedback $(1 \mathrm{RCT}, N=152)$ : no difference

PFMT + BF versus PFMT + feedback $(1 \mathrm{RCT}, N=40)$ : no difference Symptom distress:

PFMT versus PFMT + feedback + biofeedback $(1 \mathrm{RCT}, N=152):$ no difference

PFMT + BF versus PFMT + feedback ( 2 RCTs, $N=150)$ : no

difference

Pad changes in $24 \mathrm{~h}$ :

PFMT versus PFMT + feedback + biofeedback $(1 \mathrm{RCT}, N=152)$ : no difference

Adherence to treatment:

PFMT versus PFMT + feedback + biofeedback ( 1 RCT, $N=152)$ : no difference

Patients' satisfaction with progress or outcome:

PFMT + BF versus PFMT + feedback $(1 \mathrm{RCT}, N=107):$ no

difference

Global General and incontinence specific quality of life:

PFMT + BF versus PFMT (9 RCTs, $\mathrm{N}=497$ ): no difference

PFMT + BF versus PFMT + feedback (3 RCTs, $N=201)$ : no difference

Hypertension $^{22}$

$36(N=1660)^{\mathrm{c}}$

Diagnosisrelated

Blood pressure: no benefit versus pharmacotherapy. Favors sham or nonspecific behavioral interventions when combined with relaxation (unclear effect compared with behavioral or sham. confidence level: insufficient)

Secondary

Global

Intradialytic hypotension ${ }^{20}$ Diagnosis$8(N=716)^{\mathrm{d}}$
Positive

High

Positive

Medium

No effect

Low

Unsure

Insufficient $-$

All-cause mortality (2 RCTs, $N=104$ ): two deaths occurred in patients undergoing biofeedback HD, when compared with six deaths among patients undergoing conventional HD. The pooled effect estimate did not rule out a beneficial or harmful effect of biofeedback dialysis $(\mathrm{RR}=0.37,95 \% \mathrm{CI}[0.07-2.01])$
No effect Low

No effect Low

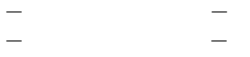

Potential Low 
Table 3. (continued)

\begin{tabular}{lccc}
\hline \hline $\begin{array}{l}\text { Condition/target } \\
\text { population }\end{array}$ & Outcomes & Findings & $\begin{array}{l}\text { Summary of } \\
\text { effect }\end{array}$ \\
$\begin{array}{l}N \text { controlled trials }(N \\
\text { combined participants) }\end{array}$ & & $\begin{array}{l}\text { Overall } \\
\text { confidence }\end{array}$ \\
\hline
\end{tabular}

Intradialytic hypotension (6 RCTs, $N=266$ ): favors biofeedback $(\mathrm{RR}=0.61,95 \%$ CI $[0.44-0.86])$

Pre-dialysis systolic blood pressure (7 RCTs, $N=203$ ): no difference $(\mathrm{MD}=3 \mathrm{mmHg}, 95 \% \mathrm{CI}[-2-7])$

Post-dialysis systolic blood pressure (3 RCTs, $N=77$ ): favors

biofeedback ( $\mathrm{MD}=7 \mathrm{mmHg}$ (95\% CI [5-19], $\left.\chi^{2}=10.52, P=0.005\right)$.

However, statistical heterogeneity may have resulted from different follow-up times and patient characteristics

Secondary Pre- and post-dialysis sodium levels (3 RCTs, $N=\mathrm{NR}$ ): no difference Urea clearance ( 3 RCTs, $N=130$ ): no difference

Post-dialysis regional wall motion abnormalities (1 RCT, $N=10)$ : favors biofeedback

Sleep bruxism ${ }^{17}$

$6(N=126)^{\mathrm{e}}$

Global

Diagnosisrelated

Quality of life (3 RCTs, $N=140$ ): mixed findings

First night's change in EMG episodes/h (3 RCTs, $N=65)$ : no

difference (MD $=-5.05,95 \%$ CI $[-10.71,0.62])$

Fifth night's change in EMG episodes/h (3 RCTs, $N=39)$ : favors

biofeedback $(\mathrm{MD}=-7.18,95 \%$ CI $[-12.54,-1.83])$

EMG activity per hour ( 2 RCTs, $N=26$ ): favors biofeedback

Secondary SB-related EMG activities $(1 \mathrm{RCT}, N=12)$ : favors biofeedback

Measurement of SB events - episodes and duration (1 RCT, $N=24)$ :

favors biofeedback

Pain (2 RCTs, $N=26$ ): no difference

Dysphagia $^{14}$

$5(N=141)$

Fibromyalgia $^{23}$

$7(N=580)$

Chronic idiopathic

constipation $^{18}$

$17(N=931)$

Knee osteoarthritis/gait retraining ${ }^{10}$

$1(N=56)$

Fecal Incontinence ${ }^{12}$

$12(N=\text { approx. } 350)^{\mathrm{g}}$

Stroke(25)

$18(N=429)$

Balance/gait training ${ }^{19}$ $8(N=243)^{\mathrm{f}}$
Global

Diagnosis-

related

Secondary

Global

Diagnosisrelated

Secondary

Global

Harms Mixed findings. Ranged from "none" to stress, fatigue, headache,

Sleep quality $(2 \mathrm{RCTs}, N=35)$ : no difference

Swallow function (2 RCTs, $N=51)$ : no difference $(\mathrm{MD}=1.10,95 \mathrm{CI}$ [-1.69-3.89])

Hyoid displacement (3 RCTs, $N=90$ ): favors biofeedback

$(\mathrm{MD}=0.22 \mathrm{~cm}, 95 \%$ CI $[0.04-0.40], P=0.02)$

Dependency on tube feeding ( 2 RCTs, $N=53)$ : no difference

$(\mathrm{OR}=3.19,95 \%$ CI $[0.16-62.72])$

$-$

Pain intensity ( 7 RCTs, $N=289$ ): favors biofeedback ( $g=0.79,95 \%$

CI $[0.22-1.36], P=0.006)$. Subgroup analyses revealed that only

EMG-BFB and not EEG-BFB significantly reduced pain intensity in comparison with control groups $(g=0.86,95 \%$ CI [0.11-1.62])

Long-term pain intensity ( 2 RCTs, $N=86)$ : no difference $(g=0.86$,

95\% CI $[-1.25-2.98], P=0.42)$

Sleep problems ( 2 RCTs, $N=87)$ : no difference $(g=0.23,95 \%$ CI [$0.20-0.65], P=0.29)$

Depression (4 RCTs, $N=181)$ : no difference $(g=0.37,95 \% \mathrm{Cl}$ [-0.44-1.18], $P=0.37$ )

Long-term depression (3 RCTs, $N=120$ ): no difference ( $g=0.8,95 \%$

CI $[-0.51-2.11], P=0.23)$

Fatigue (4 RCTs, $N=163)$ : no difference $(g=0.38,95 \% \mathrm{CI}$

[-0.46-1.08], $P=0.43$ )

Quality of life (4 RCTs, $N=163)$ : no difference $(g=0.62,95 \%$ CI [$0.77-2.02], P=0.38$ )

Long-term quality of life ( $2 \mathrm{RCTs}, N=68)$ : no difference $(g=0.252$, 95\% CI [-2.94-7.98], $P=0.37$ ) sleep problems associated with EEG and EMG biofeedback

Diagnosisrelated

Secondary

Global

Diagnosis-

related

Secondary

Global

Diagnosis-

related

Secondary

Global

Diagnosis-

related

Secondary

Global

Diagnosisrelated
Symptom management - constipation score, improved, complete spontaneous bowel movements per week: mixed findings

$-$

Pain: no difference at 3, 6, 9, and 12 months

Self-reported knee function: favors biofeedback at 3 months $(\mathrm{MD}=8.6, P=0.04)$, but not at 6 or 12 months

$-$

Remission rate (6 RCTs): favors biofeedback

Lower limb activities (17 RCTs, $N=417$ ): Favors biofeedback (SMD $0.50,95 \%$ CI $[0.30-0.70])$

$-$

Static steady-state balance outcomes:
Unclear

Insufficient

Unclear

Insufficient

Unclear

Insufficient

Unclear

Insufficient

Potential

positive

Low

No effect

Low

No effect

Low

Unclear

Insufficient

Unclear

Insufficient

$-$

Unclear

Insufficient

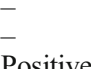

$-$

Positive Medium

- $\quad-$

Positive $\quad$ Medium

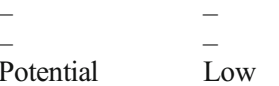


Table 3. (continued)

\begin{tabular}{|c|c|c|c|c|}
\hline $\begin{array}{l}\text { Condition/target } \\
\text { population } \\
N \text { controlled trials }(N \\
\text { combined participants) }\end{array}$ & Outcomes & Findings & $\begin{array}{l}\text { Summary of } \\
\text { effect }\end{array}$ & $\begin{array}{l}\text { Overall } \\
\text { confidence }\end{array}$ \\
\hline & & $\begin{array}{l}\text { Mediolateral_eyes open ( } 4 \text { RCTs, } N=104 \text { ): favors biofeedback } \\
\text { (Hedges' } g=0.82), 95 \% \text { CI }(0.43-1.21) \\
\text { Mediolateral_eyes closed (3 RCTs, } N=84 \text { ): favors biofeedback } \\
\text { (Hedges' } g=0.57,95 \% \text { CI [0.14-0.99]) } \\
\text { Anterior-posterior sway-eyes open: favors biofeedback (Hedges' } \\
g=0.55,95 \% \text { CI [0.01-1.10]) } \\
\text { Anterior-posterior sway eyes closed: favors biofeedback (Hedges' } \\
g=0.44,95 \% \text { CI [0.02-0.86]) } \\
\text { Dynamic steady-state balance measures: } \\
\text { Habitual gait speed: no effect (Hedges' } g=-0.19,95 \% \text { CI [ }-0.68 \text { to } \\
0.29] \text { ) }\end{array}$ & & \\
\hline & Secondary & $\begin{array}{l}\text { Studies which measured muscle strength, range of motion, and } \\
\text { physical activity did not report additional effects of WS training }\end{array}$ & Unclear & Insufficient \\
\hline & Global & Health-related quality of life: favors biofeedback & $\begin{array}{l}\text { Potential } \\
\text { Positive }\end{array}$ & Low \\
\hline \multirow[t]{2}{*}{$\begin{array}{l}\text { Raynaud's }{ }^{26} \\
10(N=531)\end{array}$} & $\begin{array}{l}\text { Diagnosis- } \\
\text { related }\end{array}$ & Symptom frequency/intensity: favors biofeedback & Unclear & Insufficient \\
\hline & Secondary & - & - & - \\
\hline \multirow[t]{3}{*}{$\begin{array}{l}\text { Labor/childbirth }{ }^{24} \\
4(N=186)\end{array}$} & $\begin{array}{l}\text { Diagnosis- } \\
\text { related }\end{array}$ & $\begin{array}{l}\text { Rates of assisted vaginal birth: no difference } \\
\text { Cesarean section: no difference } \\
\text { Augmentation of labor: no difference } \\
\text { Use of pharmacotherapy for pain: no difference }\end{array}$ & $\overline{\text { Unclear }}$ & $\overline{\text { Insufficient }}$ \\
\hline & Secondary & - & - & - \\
\hline & Global & - & - & - \\
\hline \multirow[t]{3}{*}{$\begin{array}{l}\text { Headache }{ }^{11} \\
94(N=3500+)\end{array}$} & $\begin{array}{l}\text { Diagnosis- } \\
\text { related }\end{array}$ & $\begin{array}{l}\text { Migraine reduction - frequency, duration, intensity: favors } \\
\text { biofeedback } \\
\text { Tension-type headache reduction - frequency, duration, intensity: } \\
\text { favors biofeedback }\end{array}$ & Positive & High \\
\hline & Secondary & $\begin{array}{l}\text { Medication intake: favors biofeedback } \\
\text { Muscle tension: favors biofeedback } \\
\text { Depression: favors biofeedback } \\
\text { Anxiety: favors biofeedback }\end{array}$ & Positive & Medium \\
\hline & Global & Self-efficacy: favors biofeedback & Positive & Low \\
\hline \multirow{3}{*}{$\begin{array}{l}\text { Bell's palsy }{ }^{16} \\
4(N=118)\end{array}$} & $\begin{array}{l}\text { Diagnosis- } \\
\text { related }\end{array}$ & Facial symmetry, synkinesis, lip mobility: favors biofeedback & Unclear & Insufficient \\
\hline & Secondary & - & - & - \\
\hline & Global & - & - & - \\
\hline
\end{tabular}

BF, biofeedback; $C I$, confidence interval; EEG, electroencephalograph; EMG, electromyograph; EMG, electromyogram; g, Hedge's g; MD, mean difference; P, P value; PFMT, pelvic floor muscle training; SMD, standard mean difference

${ }^{a}$ Biofeedback with pelvic floor muscle training with or without electrical stimulation

${ }^{b}$ Biofeedback with pelvic floor muscle training (PFMT) with or without feedback

${ }^{c}$ Biofeedback alone or as an adjunct versus pharmacotherapy, sham, or behavioral interventions

${ }^{d}$ Biofeedback hemodialysis versus conventional hemodialysis

${ }^{e}$ Biofeedback with swallow therapy

Only the systematic review examining fibromyalgia reported harms specifically associated with the use of biofeedback. Findings from included studies were mixed and ranged from no harm to stress associated with EMG biofeedback, and fatigue, headache, and sleep problems associated with EEG biofeedback. $^{23}$

Contributing to the low confidence levels for diagnosis-related outcomes were small combined sample sizes, poor study quality, heterogeneity in adjunctive interventions, and inconsistencies across studies included in the systematic reviews. For secondary and global outcomes, sample sizes were all less than 500 (half of those reporting secondary outcomes were less than 100), and study quality was generally poor (see Online Appendix Table 5).

\section{DISCUSSION}

This evidence map included controlled trials from 16 goodquality systematic reviews examining the effectiveness of biofeedback alone or as an adjunct for a wide range of clinical conditions. The purpose of this evidence map is to provide a high-level overview of a broad research landscape in order to illustrate the clinical conditions in which biofeedback's effectiveness is consistently supported by controlled trials, areas in which it has been shown to be ineffective, and to highlight potentially promising areas of benefit that may be good targets for more research.

We found clear, consistent evidence over a large body of trials that biofeedback can reduce the frequency, intensity, and duration of pain resulting from migraines and tension-type 
headaches (the largest reduction was in frequency); that it can also have a positive effect on headache duration and muscle tension; and that it is associated with reductions in depression, anxiety, and the need for medication. Although all biofeedback modalities included in the included review yielded medium-to-large effect sizes (EMG biofeedback with and without relaxation, EEG biofeedback, peripheral temperature biofeedback, blood volume biofeedback, and galvanic skin response), EMG biofeedback in addition to relaxation was the most effective. ${ }^{11}$ There is also clear evidence that as an adjunct to PFMT, biofeedback can provide intermediate- and long-term benefit to men experiencing urinary incontinence after a prostatectomy. ${ }^{21}$

We found consistent evidence across a smaller body of trial evidence that biofeedback is effective for attaining fecal incontinence remission. Two specific groups of patients were identified among the trials in the included systematic review-younger women who had recently given birth, and older adults of both sexes. Although no formal subgroup analyses were performed, findings in younger women were largely unclear or null. ${ }^{12}$ There is also consistent evidence in smaller trials that biofeedback is effective for short- and longterm improvements of lower limb activities after stroke. ${ }^{25}$ In the included trials, more than two-thirds of participants had experienced a stroke within the last 6 months. The review included a range of biofeedback modalities (see Table 1), and no subgroup analyses were performed. However, they did find that among studies that compared biofeedback with therapist feedback, biofeedback was superior, both clinically and statistically. Finally, larger effect sizes were reported for short-term improvements in walking than in standing, and the authors suggest that these findings may be confounded by the differences in the congruency of tools used to measure the outcomes with practiced activities. ${ }^{25}$

A smaller body of evidence suggests that the wearable sensor-based balance and gait training may be beneficial for some outcomes. Although we included a systematic review examining lower limb activities in patients after stroke, the modalities studied, outcomes examined, and patient populations differed significantly between reviews. In addition to stroke, this review also included patients with Parkinson's disease, peripheral neuropathy (due to diabetes or chemotherapy), and older adults at risk for falls. Findings suggested a positive effect on steady-state balance outcomes, but findings were mixed for outcomes related to dynamic-state balance/gait training and proactive balance training. ${ }^{19}$ A large body of smaller, largely poor-quality trials report that biofeedback (alone or combined with relaxation) is not a viable alternative to pharmacologic intervention for hypertension, ${ }^{22}$ and a metaanalysis of a similar body of evidence found that despite significantly higher ratings of patient satisfaction, biofeedback as an adjunct to PFMT provides no benefit for women experiencing urinary incontinence once differences in PFMT programs are considered. ${ }^{13}$ For other conditions, there were insufficient data to form reliable conclusions.
Few included systematic reviews included subgroup analyses by biofeedback modality or other intervention characteristics. Differences by setting (home versus center) were only reported in the systematic review examining headache, and none were found. ${ }^{11}$

To our knowledge, this evidence map is the first to examine biofeedback across a range of clinical conditions. One of the motivations for this evidence map was to identify conditions in which there is consistent evidence that biofeedback is effective. For conditions such as headache and urinary incontinence in which there is consistent data across numerous trials supporting effectiveness of biofeedback on diagnosis-related, secondary, and global health outcomes, these maps help underscore the rationale for its use in clinical practice. While the harms of biofeedback have not been well described in the literature, it is a nonpharmacologic and relatively noninvasive treatment; the risks of serious adverse effects are likely low. Given the low-risk profile of biofeedback relative to other interventions, this evidence map sought to identify conditions for which biofeedback is indicated, those for which there may be promise, and areas in need of future good-quality research.

There are a number of limitations to report, some related to evidence maps generally, and others specific to this body of research and this evidence map specifically. As the purpose of an evidence map is to provide an overview of a body of literature (and in this case, controlled trials specifically), it should not be interpreted as prescriptive, but rather as a snapshot of the evidence at a single point in time. Our rough estimates of confidence provide additional information for interpretation; however, they are not intended to replace formal evaluations of study quality or strength of evidence. We did not evaluate differences between types of biofeedback, and future systematic reviews should evaluate the effectiveness of different modalities among controlled trials. We relied on the descriptions of biofeedback modalities provided by included systematic reviews (see Table 1); thus, it is unclear whether these descriptions are consistent with AAPB-accepted terminology. We sought to include only good-quality systematic reviews, thus requiring the use of validated criteria assessing the quality/risk of bias of included studies. We also required that systematic reviews explicitly report their inclusion/ exclusion criteria and that search strategies of more than one database were both performed and reported. Many of the systematic reviews of biofeedback identified in our search did not meet these currently generally accepted criteria. For example, none of the systematic reviews we identified examining biofeedback for anxiety (identified as efficacious by the AAPB) met our inclusion criteria, nor did we include systematic reviews examining some of the other conditions for which biofeedback has been identified as potentially efficacious (e.g., alcohol/substance use, asthma, arthritis) ${ }^{27}$ or certain biofeedback modalities such as heart rate variability (HRV). Similarly, AAPB clinical guidelines are based on a series of white papers. ${ }^{11,28-36}$ Only the white paper for headache disorders met our inclusion criteria and was included in this 
evidence map. ${ }^{11}$ Finally, our inclusion of only reviews focused specifically on biofeedback for a single condition, and our focus on conditions rather than symptoms (e.g., stress) may have resulted in missed studies.

In addition to highlighting conditions for which biofeedback may be beneficial, it also identifies areas of uncertainty. Although observational studies may already suggest benefit for certain conditions not identified here, the limited number of controlled trials results in uncertainty about the true effect of biofeedback for many conditions for which it is currently recommended by the AAPB. ${ }^{27}$ We identified several conditions for which there is at least a small amount of data suggesting benefit on one or more outcomes, but in which there is not enough data to support routine clinical use. These conditions - which we identified as potentially positive on the map-include intradialytic hypotension, fibromyalgia, and balance and gait training, and these might be especially fruitful areas to prioritize further biofeedback research. Of course, we also identified numerous areas in which there was insufficient evidence to draw conclusions and these would all be areas ripe for further research. There are a number of conditions for which biofeedback is listed as efficacious by the AAPB that did not rise to the same level in our findings or were excluded due to poor systematic review methodology (i.e., urinary incontinence in females, anxiety, attention deficit hyperactivity disorder (ADHD), hypertension, and temporomandibular disorders). ${ }^{27}$ Controlled trials and good-quality systematic reviews examining these conditions are warranted. When applicable, future research should include head-to-head trials of different biofeedback modalities and settings, and when sample sizes allow, relevant subgroups should be analyzed. Finally, future systematic reviews and meta-analyses should include subgroup analyses by modality, setting, and patient characteristics; should control for heterogeneity among interventions; and aim to utilize commonly accepted AAPB terminology to describe biofeedback modalities to reduce confusion and ambiguity.

This map compliments and extends the current literature by considering only controlled trials. Many of the systematic reviews we identified included observational studies as well as trials. As a result, our conclusions may differ from the included reviews. In addition, we were conservative in our determination of effect. Biofeedback treatment protocols varied widely across studies; in instances in which subgroup analyses were performed and suggested no effect once differences were controlled, we made the determination of no effect.

\section{CONCLUSIONS}

There is clear, consistent trial evidence that biofeedback is effective in improving urinary incontinence after prostatectomy and headache; a smaller but consistent body of trial evidence also suggests biofeedback may be effective for fecal incontinence and stroke recovery. There is insufficient evidence to draw conclusions about effectiveness for many conditions, and evidence from a large number of smaller, poorer quality trials that biofeedback may be ineffective in treating urinary incontinence in women and hypertension. There are several potentially promising areas of benefit for which there is no adequate data from systematic reviews to support routine clinical use, but are good targets for further research including as follows: balance and gait training, fibromyalgia, and intradialytic hypotension.

Acknowledgments: The authors wish to thank Robin Paynter for developing the search strategy and running electronic searches and Jessica Montgomery for assisting with data abstraction.

Corresponding Author: Karli Kondo, PhD, MA; Evidence Synthesis Program, VA Portland Health Care System, 3710 SW US Veterans Hospital Road, Portland, OR 97239-2999, USA (e-mail: kondo@ohsu. edu).

Funding This research was funded by the Department of Veterans Affairs, Veterans Health Administration, Office of Research and Development, Quality Enhancement Research Initiative.

\section{Compliance with Ethical Standards:}

Conflict of Interest: The authors declare that they do not have a conflict of interest.

Disclaimer: The findings and conclusions in this document are those of the authors who are responsible for its contents; the findings and conclusions do not necessarily represent the views of the Department of Veterans Affairs or the United States government.

\section{REFERENCES}

1. Association for Applied Psychophysiology and Biofeedback. About Biofeedback [cited 2019 May 17]. Available from: https://www.aapb.org/ i4a/pages/index.cfm?pageid=3463. Accessed May 17, 2019.

2. Tan G, Shaffer F, Lyle R, Teo I. Evidence-based practice in biofeedback and neurofeedback. Third ed. Wheat Ridge: Association for Applied Psychophysiology and Biofeedback; 2016. 179 p.

3. Schwartz MS. A New Improved Universally Accepted Official Definition of Biofeedback: Where Did It Come From? Why? Who Did It? Who Is It for? What's Next? Biofeedback 2010;38(3):88-90. doi: https://doi.org/10. 5298/1081-5937-38.3.88.

4. Yucha CB, Montgomery D. Evidence-based practice in biofeedback and neurofeedback. Association for Applied Psychophysiology and Biofeedback. https://digitalscholarship.unlv.edu/nursing_fac_articles/1.

5. Miake-Lye IM, Hempel S, Shanman R, Shekelle PG. What is an evidence map? A systematic review of published evidence maps and their definitions, methods, and products. Syst Rev. 2016;5:28. doi: https:// doi.org/10.1186/s13643-016-0204-x.

6. Freeman M, Ayers C, Kondo K, Noonan K, O'Neil M, Morasco B, and Kansagara D. Guided imagery, biofeedback, and hypnosis: A map of the evidence. VA ESP Project \#05-225; 2019. Forthcoming at: https://www. hsrd.research.va.gov/publications/esp/reports.cfm.

7. Moher D, Liberati A, Tetzlaff J, Altman DG, The PRISMA Group (2009). Preferred Reporting Items for Systematic Reviews and Meta-Analyses: The PRISMA Statement. PLoS Med 6(6): e1000097. doi:https://doi.org/ 10.1371/journal.pmed1000097. Available from: www.prisma-statement. org. Accessed May 17, 2019.

8. Shea BJ, Grimshaw JM, Wells GA, Boers M, Andersson N, Hamel C, et al. Development of AMSTAR: a measurement tool to assess the methodological quality of systematic reviews. BMC Med Res Methodol. 2007;7:10. doi: https://doi.org/10.1186/1471-2288-7-10.

9. Berkman N, Lohr K, Ansari M, McDonagh M, Balk E, Whitlock E, et al. Grading the Strength of a Body of Evidence When Assessing Health Care Interventions for the Effective Health Care Program of the Agency for 
Healthcare Research and Quality: An Update. Rockville: Agency for Healthcare Research and Quality; Methods Guide for Comparative Effectiveness Reviews (AHRQ Publication No. 13(14)-EHC130-EF), 2013.

10. Richards R, van den Noort JC, Dekker J, Harlaar J. Gait Retraining With Real-Time Biofeedback to Reduce Knee Adduction Moment: Systematic Review of Effects and Methods Used. Arch Phys Med Rehabil. 2017;98(1):137-50. doi: https://doi.org/10.1016/j.apmr.2016.07.006.

11. Nestoriuc Y, Martin A, Rief W, Andrasik F. Biofeedback treatment for headache disorders: a comprehensive efficacy review. Appl Psychophysiol Biofeedback. 2008;33(3):125-40. doi: https://doi.org/10.1007/s10484008-9060-3.

12. Vonthein R, Heimerl T, Schwandner T, Ziegler A. Electrical stimulation and biofeedback for the treatment of fecal incontinence: a systematic review. Int J Colorectal Dis. 2013;28(11):1567-77. doi: https://doi.org/ 10.1007/s00384-013-1739-0.

13. Herderschee R, Hay-Smith EJC, Herbison GP, Roovers JP, Heineman MJ. Feedback or biofeedback to augment pelvic floor muscle training for urinary incontinence in women. Cochrane Database Syst Rev. 2011(7):CD009252. doi: https://doi.org/10.1002/14651858.CD009252.

14. Benfield JK, Everton LF, Bath PM, England TJ. Does therapy with biofeedback improve swallowing in adults with dysphagia? A systematic review and meta-analysis. Arch Phys Med Rehabil. 2018;100:551-61 doi: https://doi.org/10.1016/j.apmr.2018.04.031

15. Stanton R, Ada L, Dean CM, Preston E. Biofeedback improves performance in lower limb activities more than usual therapy in people following stroke: a systematic review. J Physiother. 2017;63(1):11-6. doi: https://doi.org/10.1016/j.jphys.2016.11.006.

16. Cardoso JR, Teixeira EC, Moreira MD, Favero FM, Fontes SV, Bulle de Oliveira AS. Effects of exercises on Bell's palsy: systematic review of randomized controlled trials. Otol Neurotol. 2008;29(4):557-60. doi: https://doi.org/10.1097/MAO.0b013e31816c7bf1.

17. Jokubauskas L, Baltrusaityte A. Efficacy of biofeedback therapy on sleep bruxism: A systematic review and meta-analysis. J Oral Rehabil. 2018;45:485-95. doi: https://doi.org/10.1111/joor.12628.

18. Woodward S, Norton C, Chiarelli P. Biofeedback for treatment of chronic idiopathic constipation in adults. Cochrane Database Syst Rev. 2014;3:CD008486. doi: https://doi.org/10.1002/14651858.CD008486. pub2.

19. Gordt K, Gerhardy T, Najafi B, Schwenk M. Effects of Wearable SensorBased Balance and Gait Training on Balance, Gait, and Functional Performance in Healthy and Patient Populations: A Systematic Review and Meta-Analysis of Randomized Controlled Trials. Gerontology. 2018;64(1):74-89. doi: https://doi.org/10.1159/000481454.

20. Nesrallah GE, Suri RS, Guyatt G, Mustafa RA, Walter SD, Lindsay RM, et al. Biofeedback dialysis for hypotension and hypervolemia: a systematic review and meta-analysis. Nephrol Dial Transplant. 2013;28(1):18291. doi: https://doi.org/10.1093/ndt/gfs389.

21. Hsu L-F, Liao Y-M, Lai F-C, Tsai P-S. Beneficial effects of biofeedback assisted pelvic floor muscle training in patients with urinary incontinence after radical prostatectomy: A systematic review and metaanalysis. Int $\mathrm{J}$ Nurs Stud. 2016;60:99-111. doi: https://doi.org/10.1016/j.ijnurstu. 2016.03.013.

22. Greenhalgh J, Dickson R, Dundar Y. The effects of biofeedback for the treatment of essential hypertension: a systematic review. Health technology assessment (Winchester, England). 2009;13(46):1-104. doi: https:// doi.org/10.3310/hta13460.

23. Glombiewski JA, Bernardy K, Hauser W. Efficacy of EMG- and EEGBiofeedback in Fibromyalgia Syndrome: A Meta-Analysis and a
Systematic Review of Randomized Controlled Trials. Evidence-based complementary and alternative medicine: eCAM. 2013;2013:962741. doi: https://doi.org/10.1155/2013/962741.

24. Barragan Loayza IM, Sola I, Juando Prats C. Biofeedback for pain management during labour. Cochrane Database Syst Rev. 2011(6):CD006168. doi: https://doi.org/10.1002/14651858. CD006168.pub2.

25. Stanton R, Ada L, Dean CM, Preston E. Biofeedback improves activities of the lower limb after stroke: a systematic review. J Physiother. 2011;57(3):145-55. doi: https://doi.org/10.1016/S1836-9553(11) 70035-2.

26. Karavidas MK, Tsai P-S, Yucha C, McGrady A, Lehrer PM. Thermal biofeedback for primary Raynaud's phenomenon: a review of the literature. Appl Psychophysiol Biofeedback. 2006;31(3):203-16.

27. The Association for Applied Psychology and Biofeedback. Efficacy [cited 2019 May 10]. Available from: https://www.aapb.org/i4a/pages/index. cfm?pageID=3440. Accessed May 17, 2019.

28. Template for developing guidelines for the evaluation of the clinical efficacy of psychophysiological interventions. Appl Psychophysiol Biofeedback. 2002;27(4):273-81.

29. Palsson OS, Heymen S, Whitehead WE. Biofeedback treatment for functional anorectal disorders: a comprehensive efficacy review. Appl Psychophysiol Biofeedback. 2004;29(3):153-74.

30. Monastra VJ, Lynn S, Linden M, Lubar JF, Gruzelier J, LaVaque TJ. Electroencephalographic biofeedback in the treatment of attentiondeficit/hyperactivity disorder. Appl Psychophysiol Biofeedback. 2005;30(2):95-114.

31. Crider A, Glaros AG, Gevirtz RN. Efficacy of biofeedback-based treatments for temporomandibular disorders. Appl Psychophysiol Biofeedback. 2005;30(4):333-45. doi: https://doi.org/10.1007/s10484-0058420-5.

32. Moseley JV, Linden W. The Efficacy of Behavioral Treatments for Hypertension. Appl Psychophysiol Biofeedback. 2006;31(1):51-63. doi: https://doi.org/10.1007/s10484-006-9004-8.

33. Glazer HI, Laine CD. Pelvic floor muscle biofeedback in the treatment of urinary incontinence: a literature review. Appl Psychophysiol Biofeedback. 2006;31(3):187-201. doi: https://doi.org/10.1007/s10484-0069010-x.

34. Sokhadze TM, Cannon RL, Trudeau DL. EEG biofeedback as a treatment for substance use disorders: review, rating of efficacy, and recommendations for further research. Appl Psychophysiol Biofeedback. 2008;33(1):128. doi: https://doi.org/10.1007/s10484-007-9047-5.

35. Sherman RA. White Paper: Clinical Efficacy of Psychophysiological Assessments and Biofeedback Interventions for Chronic Pain Disorders. 2004.

36. Minen MT, Azarchi S, Sobolev R, Shallcross A, Halpern A, Berk T, et al. Factors Related to Migraine Patients' Decisions to Initiate Behavioral Migraine Treatment Following a Headache Specialist's Recommendation: A Prospective Observational Study. Pain Med. 2018;19(11):2274-82. doi: https://doi.org/10.1093/pm/pny028.

Publisher's Note Springer Nature remains neutral with regard to jurisdictional claims in published maps and institutional affiliations. 